\section{Ocular syphilis: the re-establishment of an old disease}

\author{
Abstract \\ Purpose To present the patient profiles, \\ clinical presentations and visual outcomes in \\ those diagnosed with ocular syphilis, \\ attending a subspecialist uveitis service in \\ northern England over a 15-year period. \\ Patients and methods Retrospective \\ observational case series of patients \\ presenting between January 2002 and \\ December 2016.
}

Results A total of 50 eyes of 34 patients had intraocular inflammation due to syphilis. Of these, $94 \%$ were male, and $75 \%$ were men who had sex with men. Ten (29\%), all male, were HIV-positive. Presentations included isolated anterior non-granulomatous uveitis, intermediate uveitis, panuveitis, retinitis, placoid chorioretinitis and papillitis. Concurrent skin rash and/or headache were seen in $41 \%$. Compliance with antibiotic treatment was complete, but there was a high rate of default from ophthalmological followup $(38 \%)$. Visual improvement was seen in $\mathbf{9 2} \%$ of eyes, and at final assessment $71 \%$ had acuity of $6 / 9$ or better.

Conclusions Ocular syphilis is continuing to increase in incidence, in tandem with the continuing epidemic of early syphilis in the UK (the risk of ocular involvement being about $1 \%$ ). Almost all are male, and most are men who have sex with men. Clinical presentation is variable; a high index of suspicion and a low threshold for serological testing is important; early treatment can reverse retinal changes and restore visual acuity.

Eye (2018) 32, 99-103; doi:10.1038/eye.2017.155; published online 4 August 2017

\section{Introduction}

In 1999, Manchester was in the vanguard of what was to become an epidemic of syphilis throughout Europe, ${ }^{1,2}$ the disease occurring at a higher rate per head of population than any other UK centre, ${ }^{3}$ and the increase being almost
J Wells' ${ }^{1}$, C Wood ${ }^{2}$, A Sukthankar ${ }^{2}$ and NP Jones ${ }^{1,3}$

entirely in men having sex with men. ${ }^{4,5}$ As a result, ocular syphilis began to re-emerge, and the Manchester experience was described. ${ }^{6}$ Further changes in the UK incidence of syphilis ${ }^{7}$ have resulted in a continuing flow of ocular cases, permitting a re-acquaintance with the clinical spectrum of disease. We present the clinical and management data on patients with ocular syphilis diagnosed and treated in Manchester between 2002 and 2016.

\section{Patients and methods}

Patient with ocular syphilis were identified from the Manchester Uveitis Clinic database and clinical records were retrospectively reviewed. Information was cross-checked with clinical records of the Hathersage Integrated Contraception, Sexual Health \& HIV Service, with whom care was shared. The data were extracted on age, sex, sexual activity, syphilis and Human Immunodeficiency Virus (HIV) serology, ocular symptoms, signs, visual acuity (VA), and the results of treatment.

\section{Results}

Thirty-four patients developed ocular syphilis during this period, new presentations being shown in Figure 1, together with UK incidence data for early syphilis. The mean age was 41 years (range: 20-75). Thirty-two (94\%) were male, and of the 28 men whose sexual preferences were identified, 21 (75\%) were men who had sex with men (MSM). Ten patients (29\%, all male) were $\mathrm{HIV}$-positive (HIV+). Presenting symptoms included blurred vision in 23 patients, red or sore eye in 6, photopsiae in 6, central scotoma in 4 , and visual field loss in 2 . Associated systemic symptoms were identified in 14 (41\%), of whom 10 had skin rash, 4 had mucosal lesions and 5 had headache. Thirty-three of 34 patients had positive treponemal serology (treponemal agglutination test and/or ELISA) with Rapid Plasma Reagent evidence of active syphilis (titre $>1: 8$ ). The

\author{
${ }^{1}$ Manchester Royal Eye \\ Hospital, Central \\ Manchester University \\ Hospitals NHS Foundation \\ Trust, Manchester, UK

\section{${ }^{2}$ The Hathersage Integrated Contraception, Sexual Health \& HIV Service, Central Manchester University Hospitals NHS Foundation Trust, Manchester, UK}

${ }^{3}$ Medical Academic Health Science Centre, University of Manchester,

Manchester, UK

Correspondence:

NP Jones, Manchester Royal Eye Hospital, Central

Manchester University Hospitals NHS Foundation

Trust, Oxford Road,

Manchester, M13 9WL, UK Tel: +44 (0)161 2765628 . E-mail: nicholas.jones@ cmft.nhs.uk

Received: 10 January 2017 Accepted: 30 April 2017 Published online: 4 August 2017 


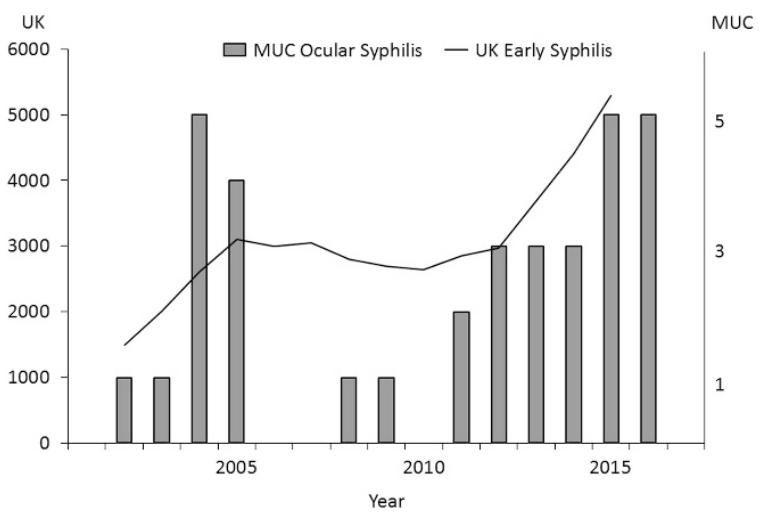

Figure 1 The incidence of new cases of ocular syphilis presenting to Manchester Uveitis Clinic (MUC) 2002-2016, compared to that of new cases of early syphilis in the UK.

remaining $\mathrm{HIV}+$ patient was PCR positive for Treponema pallidum in a vitreous sample but had negative serology and this has been previously reported. ${ }^{8}$ Three patients had previously been treated for syphilis, no episodes involving the eye. One patient presented with visual symptoms having just commenced doxycycline treatment for secondary syphilis; in the remainder (97\%), presentation was with visual symptoms, and ocular signs provoked serology leading to diagnosis.

Of the 34 patients, 16 (47\%) had bilateral uveitis. In the remaining 18, the right eye was involved in 6 cases and the left in 12. This difference is somewhat significant $(P=0.02)$. Seven of $10 \mathrm{HIV}+$ patients had unilateral involvement (all L-sided), the remaining three bilateral. Of the 50 affected eyes, 3 had non-granulomatous anterior uveitis alone (all HIV+), 7 had intermediate-type uveitis $(0 \mathrm{HIV}+)$ and 24 had panuveitis (5 HIV+). Retinitis was seen in 25 ( $3 \mathrm{HIV}+)$, of which one was necrotising and developed retinal detachment; 6 had macular placoid chorioretinitis $(0 \mathrm{HIV}+)$, and 14 had papillitis (3 $\mathrm{HIV}+)$. One patient had focal scleritis overlying an area of retinitis. Two had significant retinal vasculitis $(0 \mathrm{HIV}+)$.

Twenty-seven patients completed treatment in accordance with the UK national guidelines on the management of neurosyphilis 2008 (17 consecutive days of intramuscular procaine penicillin $2.4 \mathrm{MU}$ with oral probenecid $500 \mathrm{mg} \mathrm{QID}{ }^{9}$ ) and 4 patients were treated using the 2015 updated guidelines, ${ }^{10}$ which recommended 14 rather than 17 days of penicillin (other treatment being unchanged). Treatment was not altered for HIV positivity. One patient refused intramuscular treatment and another was allergic to penicillin; both were treated with intravenous ceftriaxone for 10 days. One patient refused all treatment despite counselling and defaulted from follow-up. All patients treated with antibiotic received at least 3 days of oral prednisolone commencing one day prior to antibiotic treatment to

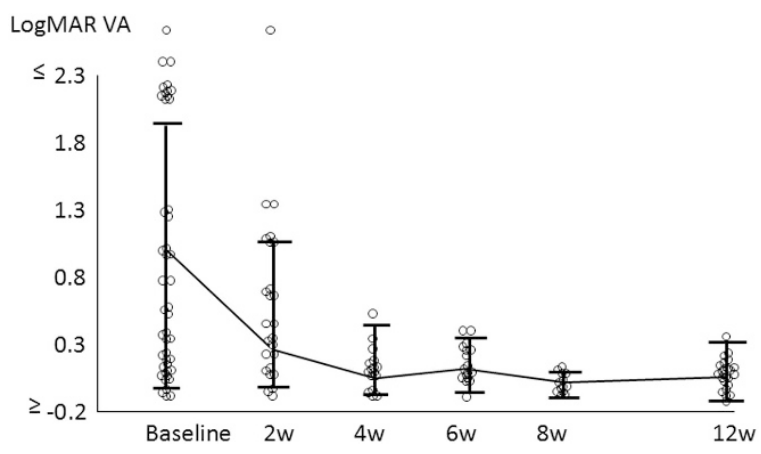

Figure 2 Baseline and subsequent LogMAR visual acuities for 50 eyes treated for syphilitic uveitis, means and $95 \%$ confidence intervals shown.

prevent Jarish-Herxheimer reactions, and no intraocular inflammation flares were witnessed. Twenty patients were felt to require more prolonged oral steroid treatment for their uveitis, commencing at 25-60 mg per day for 4-12 weeks. There was no evidence that HIV positivity worsened visual outcome.

Thirteen of 34 patients ( $38 \%$ ) defaulted from follow-up (including 5 out of $10 \mathrm{HIV}+$ patients). Of those patients who completed follow-up and were discharged, the duration of follow-up ranged from 8 weeks to 11 months (one patient is still being reviewed 5 years following treatment due to co-existent pathologies).

The visual acuity at presentation and thereafter in affected eyes is shown in Figure 2 (including mean and 95\% CI VA at intervals). At presentation, 32 eyes (64\%) had VA worse than 6/9. Two patients defaulted from all ophthalmological follow-up and there are no data on visual result. Visual improvement or recovery occurred in 46 out of 50 eyes, and at final assessment (of variable period from presentation) 34 eyes (71\%) had acuity of $6 / 9$ or better, and 14 eyes (28\%) 6/6 or better.

\section{Discussion}

The syphilis epidemic first reported in Manchester in $1999^{1,2}$ and subsequently affecting most cities in Europe and beyond led to a progressively increasing incidence of new cases of early syphilis until a peak in 2005. A temporary decline in incidence followed, but then a subsequent further rise so that between 2010 and 2014, Public Health England recorded a $40 \%$ rise in incidence of all syphilis. ${ }^{11}$ In 2015, the number of new cases of early syphilis reported in the UK was 5288, the highest rate for over 70 years. ${ }^{11}$ These incidence changes have been reflected fairly accurately by the number of new cases of syphilitic uveitis (Figure 1). The demography of Manchester and its ophthalmological referral patterns, together with a knowledge of reported cases of early 
syphilis and uveitis allow us to estimate a risk of about $1 \%$ for ocular involvement in those with early syphilis.

In this group of patients, where intraocular inflammation was unilateral, the left eye was significantly more likely to be affected $(P=0.02)$. The same phenomenon has previously been noted in a UK national survey. ${ }^{12}$ A previous study from this centre ${ }^{13}$ found that several forms of uveitis tended to favour the right side (Cytomegalovirus retinitis, fungal or bacterial endophthalmitis, toxocariasis and toxoplasmosis) but none (including syphilis at that stage) favoured the left. The cause of this phenomenon, if genuine, is unknown.

The most frequent form of intraocular inflammation in this series was panuveitis, seen in $48 \%$ of eyes. Overall, the posterior segment was inflamed in $90 \%$ of eyes; This tendency has previously been reported. ${ }^{14,15}$ It is clear that isolated anterior uveitis is rare in this disease; both affected patients here were HIV-positive. This also reflects a previous literature review ${ }^{16}$ which found that $96 \%$ of patients presenting with isolated anterior uveitis were HIV positive, giving a relative risk of HIV positivity in syphilitic isolated anterior uveitis of 14.5. HIV co-infection in our study was relatively low at $29 \%$. Others have reported rates between $21 \& 83 \%, 12,14-17$ with lower frequencies in Asian case series (21-24\%) $)^{15,17}$ than in Western countries.

The VA at presentation was widely variable (Figure 2) and this reflected the presence or absence of direct macular involvement or the severity of panuveitis. Visual improvement or complete recovery occurred in $92 \%$ of eyes supervised. However, visual results should be interpreted with caution; despite a very high rate of compliance with antibiotic treatment, there was a high tendency (38\%) to default from ophthalmological followup at varying stages, and 5 affected eyes had VA of 6/60 or worse before default. Those defaulting tended to have poorer visual acuity at their last recorded examination, than those who did not. Patients who were HIV+ were somewhat more likely to default. Bearing these limitations in mind, we nevertheless did not observe any significant difference in visual outcome between HIV+ and HIV- patients. This supports the current National guidelines which recommend the same antibiotic regimen for HIV+ and HIV- patients.

Acute syphilitic posterior placoid chorioretinitis (ASPPC) is an increasingly recognised presentation of

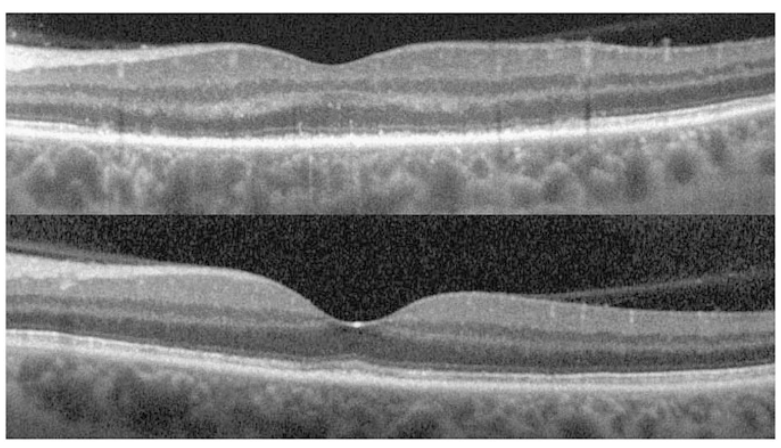

Figure 4 Spectral-domain optical coherence tomography images of acute syphilitic posterior placoid chorioretinitis (top, visual acuity 6/18), demonstrating typical hyper-reflective nodular retinal pigment epithelial thickening and disruption of outer segment. Three months after treatment (bottom) the scan is anatomically normal (visual acuity 6/5).

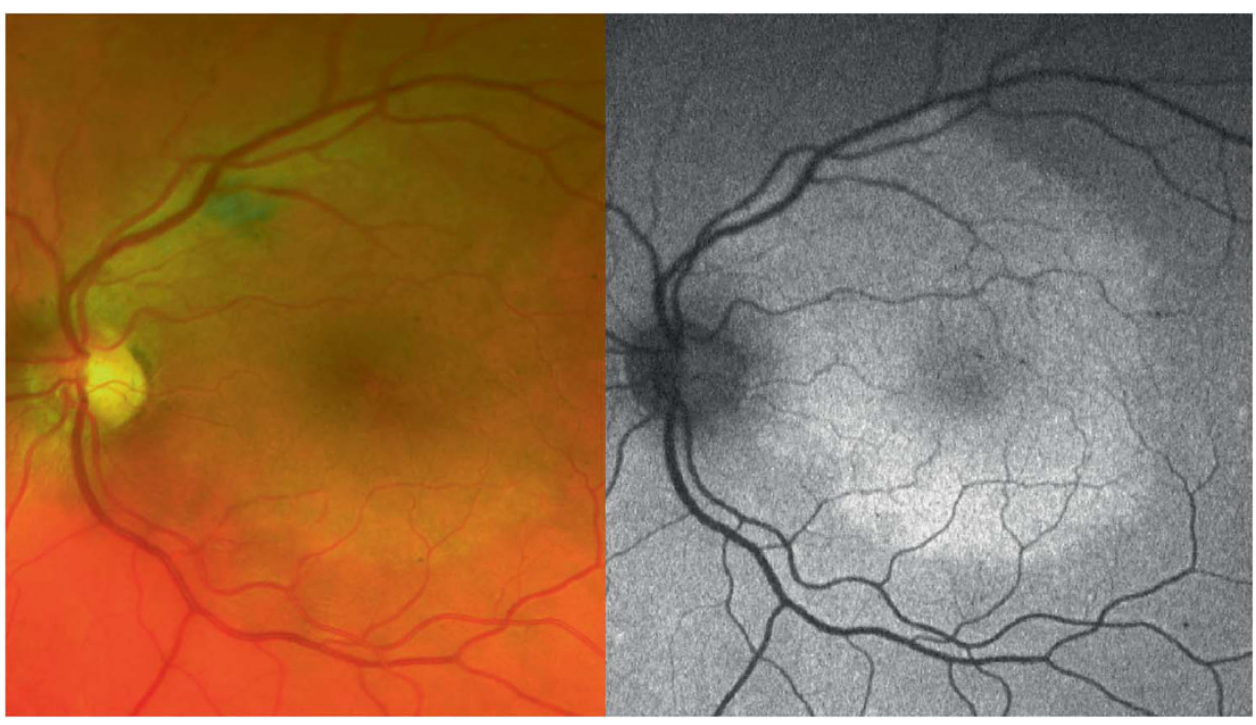

Figure 3 Early acute syphilitic posterior placoid chorioretinitis, shown by Optomap false-colour (left) and autofluorescence (right). The lesion was virtually undetectable on clinical examination. 


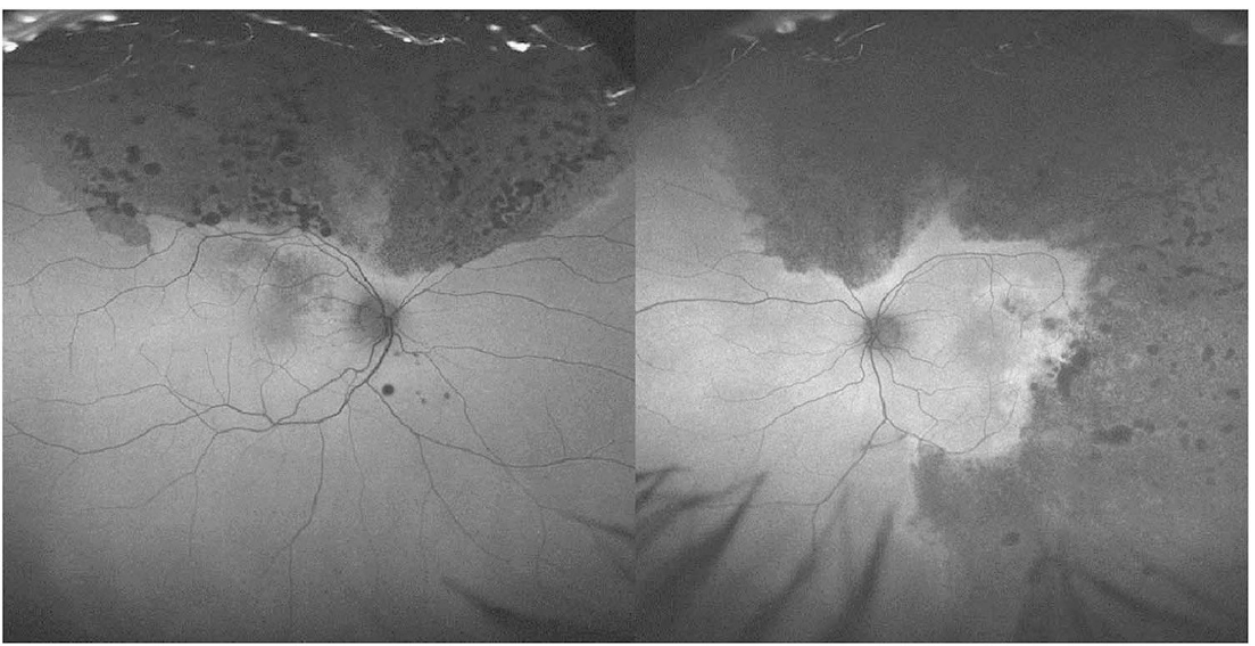

Figure 5 Widespread scarring following syphilitic retinitis shown by ultra-widefield autofluorescence, leading to relative nyctalopia.

syphilitic ocular disease and was seen in 6 eyes $(12 \%)$ in this series. Its early appearance can be very subtle on clinical examination, but we have found that false-colour images obtained using red/green laser systems (such as the Optos fundus camera) enhances contrast of the placoid lesion, aiding in diagnosis (Figure 3a), as does autofluorescence $^{18}$ (Figure 3b). Placoid lesions are characteristic on OCT imaging with acute RPE thickening, hyper-reflectivity and subretinal fluid, followed by RPE irregularity and disruption of the outer retinal layers (Figure $4 \mathrm{a}$ ) and we, like others ${ }^{19}$ have recorded complete OCT reversal of signs (Figure $4 \mathrm{~b}$ ) with early treatment followed by excellent visual outcome. In contrast, latepresenting ASPPC leads to permanent scarring as recorded previously. ${ }^{20,21}$ Our patients with ASPPC were all HIV-negative. Early suggestions that ASPPC was more likely in HIV+ patients does not appear to have been supported by wider analysis. ${ }^{22}$ Spreading retinitis may occur in those diagnosed late; despite treatment, large areas of non-functioning retina may result (Figure 5) and two patients were left with relative nyctalopia.

Papillitis was seen in 14 eyes of 10 patients in this series, of whom only two had concurrent headache. Nine of these 14 eyes also had panuveitis. Papillitis was most frequently present in eyes with panuveitis or retinitis, but also occurred in isolation, unilaterally in one patient. Papillitis was not associated with a poorer visual outcome, in contrast to a previous report. ${ }^{23}$

In conclusion, syphilitic uveitis is rising in incidence in tandem with a continuing overall rise in incidence of early syphilis in the UK; ocular syphilis affects about $1 \%$ of those with early syphilis. Inflammation can present with variable features, the most striking being zonal retinitis or macular placoid retinopathy. A high level of vigilance in undertaking relevant history-taking for associated risk factors and symptoms is important, with a low threshold for investigation in new patients with suspicious uveitis; early diagnosis and treatment usually leads to a good visual outcome.

\section{Summary}

What was known before

- Ocular syphilis is rare and can blind Intraocular involvement is protean.

What this study adds

- Ocular syphilis is much more common and increasing in incidence Retinitis is characteristic and sometimes pathognomonic Early diagnosis and treatment can repair retinal involvement.

\section{Conflict of interest}

The authors declare no conflict of interest.

\section{Acknowledgements}

This research was facilitated by the Greater Manchester Local Clinical Research Network.

\section{References}

1 Higgins SP, Sukthankar A, Mahto M, Jarvis RR, Lacey HB. Syphilis increases in Manchester, UK. Lancet 2000; 355: 1466.

2 Lacey HB, Higgins SP, Graham D. An outbreak of early syphilis: cases from North Manchester General Hospital. Sex Transm Infect 2001; 77: 311-313.

3 Righarts AA, Simms I, Wallace L, Solomou M, Fenton KA. Syphilis surveillance and epidemiology in the United Kingdom. Euro Surveill 2004; 9: 21-25. 
4 Kingston MA, Higgins SP. Audit of the management of early syphilis at North Manchester General Hospital. Int J STD AIDS 2004; 15: 352-354.

5 Ashton M, Sopwith W, Clark P, McKelvey D, Lighton L, Mandal. D. An outbreak no longer: factors contributing to the return of syphilis in Greater Manchester. Sex Transm Infect 2003; 79: 291-293.

6 Doris J, Saha K, Jones NP, Sukthankar A. Ocular Syphilis the new epidemic. Eye 2006; 20: 703-705.

7 Anon 2013. Recent epidemiology of infectious syphilis in England. Infection Reports, vol 7, Public Health England: London, UK. November 2013, pp 44.

8 Troutbeck R, Chhabra R, Jones NP. Polymerase chain reaction testing of vitreous in atypical ocular syphilis. Ocul Immunol Inflamm 2013; 21: 227-230.

9 Kingston M, French P, Goh B, Goold P, Higgins S, Sukthankar ASyphilis Guidelines Revision Group 2008. UK National guidelines on the management of syphilis 2008. Int J STD AIDS 2008; 19: 729-740.

10 Kingston M, French P, Higgins S, McQuillan O, Sukthankar A, Stott C Syphilis Guidelines Revision Group 2015 et al. UK National guidelines on the management of syphilis 2015. Int J STD AIDS 2016; 27: 421-446.

11 Anon 2016. Health protection report, vol 10. Public Health England: London, UK, pp 22.

12 Mathew RG, Goh BT, Westcott MC. British Ocular Syphilis Study (BOSS): 2-year national surveillance study of intraocular inflammation secondary to ocular syphilis. Invest Ophthalmol Vis Sci 2014; 55: 5394-5400.

13 Jones NP. Manchester Uveitis Clinic: the first 3000 patients: 2. complications and management. Ocul Immunol Inflamm 2015; 23: 127-134.

14 Northey LC, Skalicky SE, Gurbaxani A, McCluskey PJ. Syphilitic uveitis and optic neuritis in Sydney, Australia. Br J Ophthalmol 2015; 99: 1215-1219.
15 Yang P, Zhang N, Li F, Chen Y, Kijlstra A. Ocular manifestations of syphilitic uveitis in Chinese patients. Retina 2012; 32: 1906-1914.

16 Amaratunge BC, Camuglia JE, Hall AJ. Syphilitic uveitis: A review of clinical manifestations and treatment outcomes of syphilitic uveitis in human immunodeficiency virus-positive and negative patients. Clin Exp Ophthalmol 2010; 28: 68-74.

17 Yap SC, Tan YL, Chio MTW, Teoh SC. Syphilitic uveitis in a Singaporean population. Ocul Immunol Inflamm 2014; 22(1): 9-14.

18 Matsumoto Y, Spaide RF. Autofluorescence imaging of acute syphilitic posterior placoid chorioretinitis. Retin Cases Brief Rep 2007; 1: 123-127.

19 Pichi F, Ciardella AP, Cunningham Jr ET, Morara M, Veronese C, Jumper JM et al. Spectral domain optical coherence tomography findings in patients with acute syphilitic posterior placoid chorioretinopathy. Retina 2014; 34: 373-384.

20 Armstrong BK, Pitcher J, Shah R, Brady C, Perlmutter D, Garg SJ. The evolution of untreated acute syphilitic posterior placoid chorioretinitis captured by multimodal retinal imaging. Ophthalmic Surg Lasers Imaging Retina 2014; 45: 606-609.

21 Moradi A, Salek S, Daniel E, Gangaputra S, Ostheimer TA, Burkholder BM et al. Clinical features and incidence rates of ocular complications in patients with ocular syphilis. Am J Ophth 2015; 159(2): 334-343.

22 Eandi CM, Neri P, Adelman RA, Yannuzzi LA, Cunningham ET Jr. International Syphilis Study Group. Acute syphilitic posterior placoid chorioretinitis: report of a case series and comprehensive review of the literature. Retina 2012; 32: 1915-1941.

23 Balaskas K, Sergentanis TN, Giulieri S, Guex-Crosier Y. Analysis of significant factors influencing visual acuity in ocular syphilis. Br J Ophthalmol 2011; 95: 1568-1572. 